\title{
Thermal cycling and the optical and electrical characterization of self-assembled multilayer Nile Blue A-gold thin films
}

\author{
Brian Geist, William B. Spillman, Jr., and Richard O. Claus
}

\begin{abstract}
Some laser applications produce high power densities that can be dangerous to equipment and operators. We have fabricated thin-film coatings by using molecular electrostatic self-assembly to create a spectrally selective absorbing coating that is able to withstand thermal fluctuations from $-20{ }^{\circ} \mathrm{C}$ to $120^{\circ} \mathrm{C}$. We made the thin-film coatings by alternating deposition of an organic dye and gold colloidal nanoparticles onto glass substrates. Nile Blue A perchlorate, with a maximum absorbance slightly above $632 \mathrm{~nm}$, was chosen as the organic dye. Strong coupling between the dye molecules and the gold nanoparticles provides a redshift that increases as the film's thickness is increased. The incorporation of the gold colloidal nanoparticles also decreases the resistivity of the film. The resistivity of the film was measured with a four-point probe and found to be $\sim 10 \Omega / \mathrm{cm}$ for the two samples measured. Atomic-force microscopy was used to show that film thickness increased $2.4 \mathrm{~nm}$ per bilayer. The optical properties of the film were measured at the end of every 5 thermal cycles from $-20{ }^{\circ} \mathrm{C}$ to $120^{\circ} \mathrm{C}$, and negligible degradation was observed after 30 cycles. (C) 2005 Optical Society of America
\end{abstract}

OCIS codes: $\quad 230.4170,310.1620,310.1860,310.3840,310.6860,350.1820$.

\section{Introduction}

The bulk properties of organic dye molecules have been investigated by experimental examination of spin-coated dyes on metal films. ${ }^{1}$ Recently, nanoscale molecular electrostatically self-assembled (ESA) films, consisting of alternating layers of metallic colloidal nanoparticles coated in combination with a polyelectrolyte and polymer dyes, were shown to exhibit conductivities similar to that of bulk metal. ${ }^{2}$ Further studies indicated that such films show an enhancement of optical absorption at specific wavelengths owing to a strong resonance interaction between the metallic nanoparticles and the dye molecules. ${ }^{3}$ Widely different electrodeposition techniques have been used to construct oligothiophenelinked metallic nanoparticles by use of organic bridging molecules. ${ }^{4,5}$ Additionally, polystyrene

The authors are with Virginia Polytechnic Institute and State University, Blacksburg, Virginia 24061. B. Geist (bgeist@vt.edu) and W. B. Spillman, Jr., are with the Applied Biosciences Center and R. O. Claus is with the Fiber \& Electro-Optics Research Center.

Received 24 March 2005; revised manuscript received 17 May 2005; accepted 24 May 2005.

$0003-6935 / 05 / 306357-04 \$ 15.00 / 0$

(C) 2005 Optical Society of America spheres have been used as templates for metallic nanoparticle adsorption, making it possible to tune the optical properties of a film by controlling particle sizes and densities. ${ }^{6}$ ESA has been used to attach fullerenes to the metallic nanoparticles, which allowed for further tuning of the optical properties. ${ }^{7}$

It is important to evaluate the survivability of these ultrathin films in changing environments to determine any limitations that might hamper their widespread use. In this study, we have conducted thermal cycling of ESA films. Fabricated samples were exposed to temperature fluctuations, which were thought to potentially cause either thermal breakdown or mechanical failure because of the different thermal expansion rates of the individual monolayers or of the multilayer stack and the substrate.

In our experiments ESA was used to form alternating layers of Nile Blue A perchlorate and gold upon glass substrates. Nile Blue A perchlorate, an organic dye, was chosen for one of the monolayers whose peak absorbance wavelength is slightly higher than $632 \mathrm{~nm}$. The other alternating monolayer was fabricated of gold colloidal nanoparticles. The use of goldnanoparticles decreased the resistivity of the film and provided a different type of material with which to test the effects of thermal cycling. ${ }^{2}$ The bilayers resulted in a high optical-absorption-to-thickness ratio. 


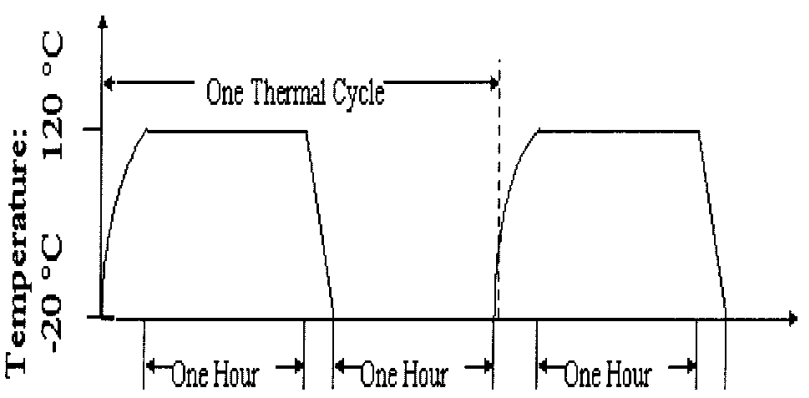

Fig. 1. Thermal cycling process used to test the Nile Blue A perchlorate-gold film.

The ESA process produced a high film uniformity that ensured homogeneous absorbance spectra across the surface of the film. ${ }^{8}$ The process can easily be adapted to form bilayers on any surface geometry at room temperature and at atmospheric pressure. In this paper we present the results of temperature degradation studies that we carried out to determine the survivability of the films over many thermal cycles from $-20{ }^{\circ} \mathrm{C}$ to $120^{\circ} \mathrm{C}$ in ambient atmospheric conditions.

\section{Experimental Procedure}

Sample materials were obtained from Aldrich. Nile Blue A perchlorate came in powder form and was water soluble. A concentration of $5 \mathrm{mM}$ of Nile Blue A perchlorate in deionized water was used throughout the experiment. A solution of gold colloids was made by addition of $1 \mathrm{~mL}$ of a $24.3 \mathrm{mM} \mathrm{HAuCl}{ }_{4}$ solution of deionized water dropwise into $106 \mathrm{~mL}$ of a rapidly boiling $2.2 \mathrm{mM}$ sodium citrate solution in deionized water. The chemical compositions by weight of the resultant compounds, in atomic mass units, were as follows: Nile Blue A perchlorate, $240 \mathrm{C}, 20 \mathrm{H}, 35.45$ $\mathrm{Cl}, 42 \mathrm{~N}$, and $80 \mathrm{O}$; gold colloids, $197 \mathrm{Au}$.

Glass substrates were prepared by bathing in a 7:3 mixture of sulfuric acid and hydrogen peroxide for $30 \mathrm{~min}$. The acid wash was used to draw a negative charge to the surface of the substrate, and the hydrogen peroxide was used to clean the substrate. Nitrogen gas was then used to dry the surface of the substrate. The films were constructed by use of a

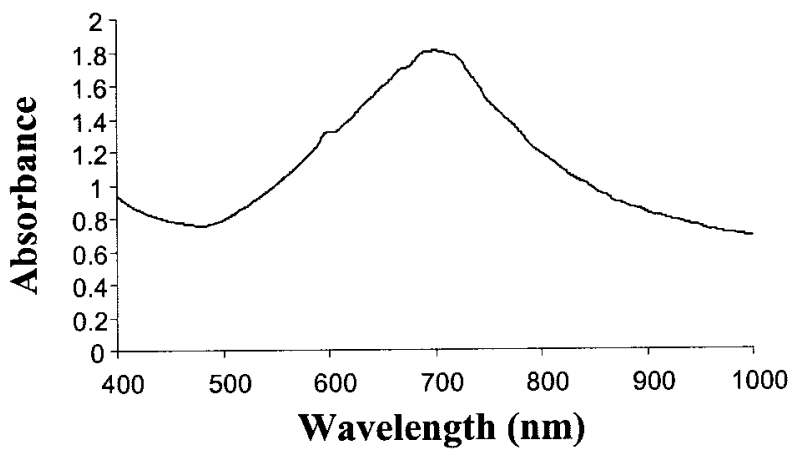

Fig. 2. Absorbance of an unheated Nile Blue A perchlorate-gold 30 bilayer sample.

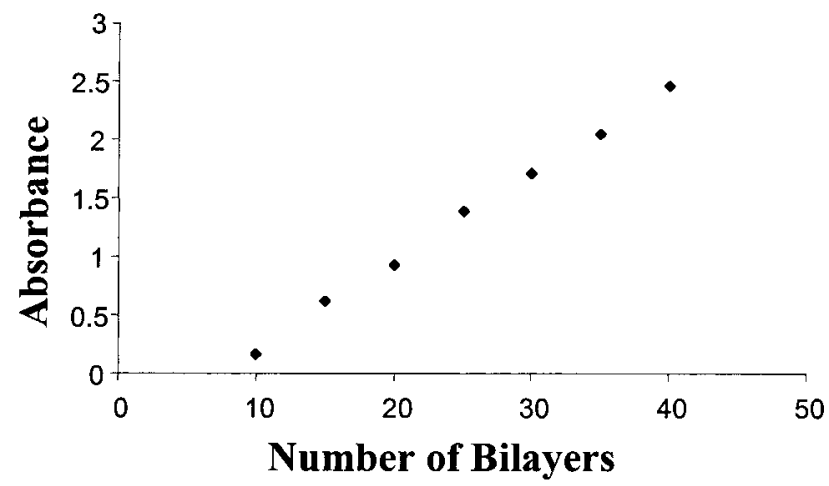

Fig. 3. Maximum absorbance of a Nile Blue A perchlorate-gold film.

StratoSequence V dipping machine. The dipping machine dipped the substrate into a solution of positively charged Nile Blue A perchlorate molecules for 5 min. During this time, the Nile Blue A perchlorate molecules were adsorbed to the surface of the substrate, leaving a net positive charge on the surface. The dipping machine then rinsed the sample with deionized water to remove loosely bound molecules. The dipping machine then dipped the substrate into a solution of negatively charged gold nanoparticles for $5 \mathrm{~min}$. Further rinsing with deionized water was performed to remove any loosely adsorbed gold nanoparticles. The result of this process was one bilayer of Nile Blue A perchlorate-gold with a negatively charged surface. During the building process, a Hitachi U-2001 ultraviolet-visible spectrophotometer was used to measure the absorbance from 400 to $1000 \mathrm{~nm}$ every 5 bilayers up to 40 bilayers.

Thermal cycling was performed in a programmable ESPEC SH-240 temperature and humidity chamber with temperatures ranging from $-20^{\circ} \mathrm{C}$ to $120^{\circ} \mathrm{C}$ on samples of 10,20 , and 30 bilayers to assess the survivability of the thin films in changing environments. The samples were held at each of the temperature extremes for $1 \mathrm{~h}$ per cycle in ambient atmospheric conditions, as shown in Fig. 1. We used the UVvisible spectrophotometer at the end of every five

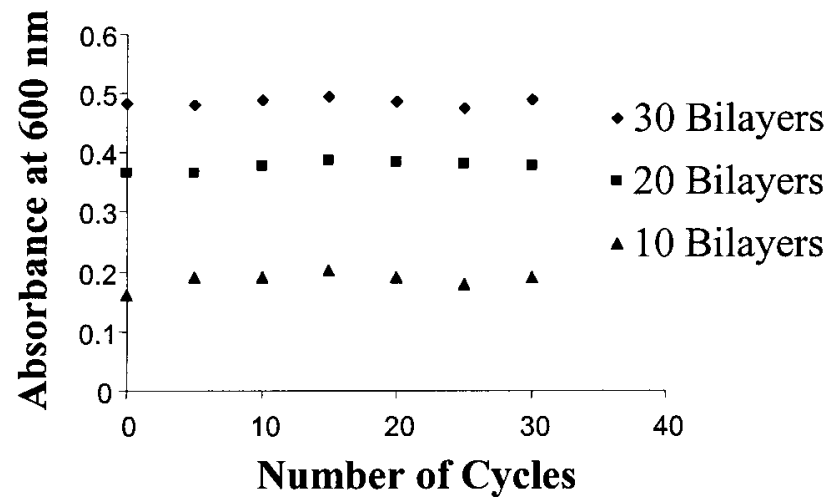

Fig. 4. Thermal cycling effects on a Nile Blue A perchlorate-gold film $\left(-20^{\circ}\right.$ to $\left.120^{\circ} \mathrm{C}\right)$. 


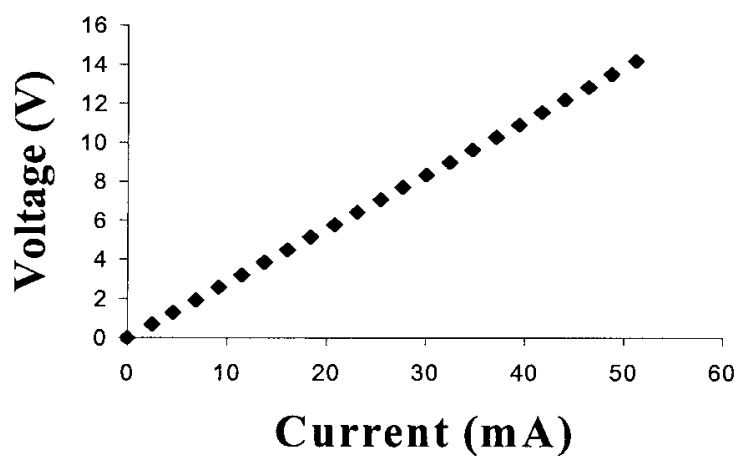

Fig. 5. Voltage versus current for a 50 bilayer Nile Blue A perchlorate-gold film.

thermal cycles to determine whether thermal cycling had any effects on the optical quality of the film.

Resistivity measurements were conducted on samples of 50 and 100 bilayers. An Edwards FL 400 thermal evaporator was used to deposit four aluminum contact pads onto the sample. An Agilent E3630A dc power supply was used to provide current through the sample. Two Agilent 34401A 61/2 digit multimeters were used to measure the current through and the voltage across the sample. Atomicforce microscopy (AFM) was performed to produce a topographical image of the sample by use of a Dimension 3100 AFM from Digital Instruments to determine film thickness by measuring the height difference between the surface of the film and the substrate at the film edge. A caliper was used to determine the length and width of the film. With the instruments mentioned above, we used a four-point probe measuring system following the method described in Standard A712-75 from the American Society for Testing and Materials ${ }^{9}$ to measure sample resistivity. The sample was measured in the forward and reverse current directions. Voltage was plotted against current for both cases, with the slope of the plots providing the resistance. The formula $R_{\text {actual }}$ $=\left(R_{\text {forward }}-R_{\text {reverse }}\right) / 2$ was used to account for any offset voltages that might occur. We used $R_{\text {actual }}$ and

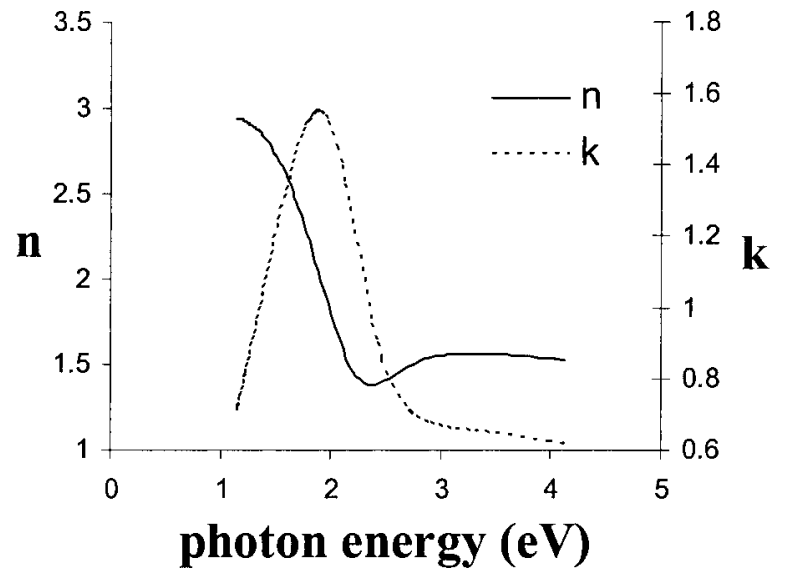

Fig. 6. Index of refraction and extinction coefficient of a Nile Blue A perchlorate-gold film.

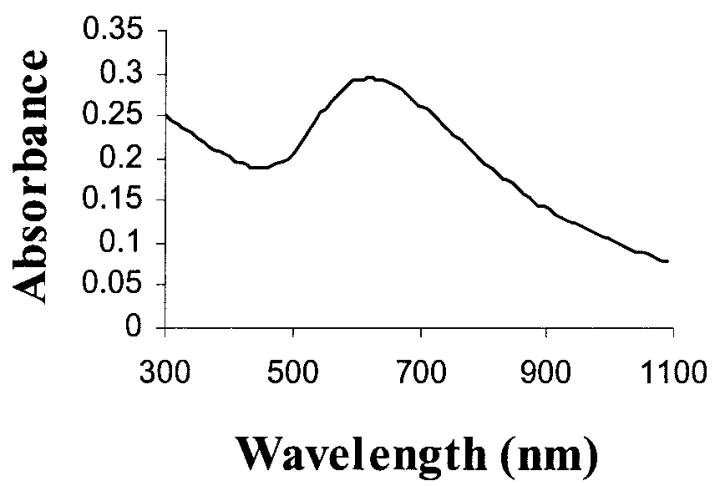

Fig. 7. Absorbance versus wavelength calculated from the extinction coefficient.

the dimensions measured with the AFM and the caliper to calculate the resistivity with the formula $\rho=$ (width $\times$ thickness $\times R_{\text {actual }} /$ length) to obtain the resistivity. A Woollam variable-angle spectroscopic ellipsometer was used to determine the index of refraction and the extinction coefficient of the film by use of the thickness obtained by the AFM and the modeling software provided by Woollam with the ellipsometer.

\section{Results}

The ESA process using Nile Blue A perchlorate-gold was shown to produce a high ratio of absorbance to thickness. The absorbance spectrum indicates that the maximum absorption is $\sim 700 \mathrm{~nm}$, as shown in Fig. 2. The bilayers provide the individual dye molecules with direct contact to gold nanoparticles, giving a greatly increased absorbance owing to surfaceenhancement phenomena. ${ }^{3}$ The absorption enhancement is due to a charge-transfer mechanism where by electronic excitation may be transferred between the dye molecule and the metallic nanocluster. As more bilayers are built, a slight redshift in maximum absorbance is observed, indicating a greater number of charge transfers through the ionic bands of the dye molecules and gold nanoclusters. ${ }^{1}$ The charge transfer results in a redshift because the plasma frequency of the gold nanoparticles has a corresponding wavelength that is greater than the absorbance maximum of the Nile Blue A perchlorate molecules. A redshift of the maximum absorbance of the film occurs as a function of thickness and shifts the maximum absorbance located at $680 \mathrm{~nm}$ for 5 bilayers to $\sim 710$ for 40 bilayers. Figure 3 shows that the growth in absorbance for the first ten bilayers is less than the absorbance growth rate after ten bilayers have been deposited, suggesting that the coverage of the bilayers on the substrate was not complete until after ten bilayers had been formed. The growth in maximum absorbance is linear after the first 10 bilayers are formed, indicating that the ESA process does not break down for as many as 40 bilayers. The absorbance for larger numbers of bilayers was greater than could be determined by the spectrophotometer.

Thermally cycled samples of 10,20 , and 30 bilayers 
displayed negligible degradation from temperatures up to $120^{\circ} \mathrm{C}$. Figure 4 shows that the three samples maintain constant UV-visible absorption characteristics. The absorbance of the samples was measured at the end of every five thermal cycles. It is interesting to note that the thickness of the films had no effect on film degradation throughout the cycling process.

AFM in tapping mode found the film thickness to be $\sim 2.4 \mathrm{~nm}$ per bilayer for the 50 and 100 bilayer films. Resistivity was the calculated to be $\sim 10.4 \Omega$ / $\mathrm{cm}$ for a 50 bilayer film and $9.3 \Omega / \mathrm{cm}$ for a $100 \mathrm{bi}-$ layer film. Figure 5 shows that the material displays a linear increase in voltage as the current is increased. This linear increase suggests that the film behaves as an ohmic resistance.

The index of refraction and the extinction coefficient for the thin film exhibit ultrathin metallike behavior. ${ }^{10}$ Figure 6 shows that a ten bilayer film has a high index of refraction in the optical range that decreases to approximately that of glass in the near IR range. We converted extinction coefficient $k$ shown in Fig. 6 to absorbance and plotted it versus wavelength as shown in Fig. 7. A blueshift is seen in Fig. 7 compared with Fig. 2, which can be partially attributed to the fact that the data shown in Fig. 7 come from a 10 bilayer film whereas the data shown in Fig. 2 come from a 30 bilayer film.

\section{Discussion and Summary}

Nile Blue A perchlorate-gold ESA thin films have shown linear growth in absorbance as a function of bilayer number. Therefore, with this method it is relatively easy to build a film with the desired absorbance. When we built the films it was found necessary always to use a freshly made gold colloid solution. The gold colloid particles were found to be highly susceptible to impurities and to possible chemical reactions caused by the $\mathrm{pH}$ level of the rinse water, and the Nile Blue A perchlorate solution sometimes causes the gold colloid particles to fall out of solution. The films showed acceptable durability in the thermal cycling. Further tests may be performed to determine the film breakdown temperature.

This project was made possible by Tim Peterson and David Zelmon of the U.S. Air Force Research Laboratories, Wright-Pattérson Air Force Base, who provided advice on the characterization techniques used on these ESA films. The authors are grateful to Kurt Eyink, also of the U.S. Air Force Research Laboratories, who performed the ellipsometry measurements.

\section{References}

1. A. M. Glass, P. F. Liao, J. G. Bergman, and D. H. Olson, "Interaction of metal particles with adsorbed dye molecules: absorption and luminescence," Opt. Lett. 5, 368-370 (1980).

2. Y. Liu, Y.-X. Wang, and R. O. Claus, "Layer-by-layer ionic self-assembly of Au-colloids into multilayer thin-films with bulk metal conductivity," Chem. Phys. Lett. 298, 315-319 (1998).

3. Y. Liu and R. O. Claus, Strong Enhancement of Optical Absorbance from Ionic Self-Assembled Multilayer Thin Films of Nanocluster Pt and Polymer Dye, J. Appl. Phys. 85, 419-424 (1999).

4. B. C. Sih, A. Teichert, and M. O. Wolf, "Electrodeposition of oligothiophene-linked gold nanoparticle films," Chem. Mater. 16, 2712-2718 (2004).

5. S. Chen, "Self-assembling of monolayer-protected gold nanoparticles," J. Phys. Chem. B 104, 663-667 (2000).

6. H. Takei, "Surface-adsorbed polystyrene spheres as a template for nanosized metal particle formation: optical properties of a nanosized Au particle," J. Vac. Sci. Technol. B 17, 1906-1911 (1999).

7. H. Fang, C. Du, S. Qu, Y. Li, Y. Song, H. Li, H. Liu, and D. Zhu, "Self-assembly of the [60]fullerene-substituted oligopyridines on Au nanoparticles and the optical nonlinearities of the nanoparticles," Chem. Phys. Lett. 364, 290-296 (2002).

8. G. Decher and J. B. Schlenoff, Multilayers Thin FilmsSequential Assembly of Nanocomposite Materials (Wiley-VCH, 2003).

9. American Society for Testing and Materials, Standard Test Method for Electrical Resistivity of Soft Magnetic Alloys, Standard A712-75 (American Society for Testing and Materials, 1991).

10. S. Y. Park and D. Stroud, "Structure formation, melting, and optical properties of gold/DNA nanocomposites: effects of relaxation time," Phys. Rev. B 68, 224201 (2003). 